\title{
Explicit boundary control of a reaction-diffusion equation on a disk
}

\author{
Rafael Vazquez* Miroslav Krstic ${ }^{* *}$ \\ * Dept. of Aerospace Engineering, Universidad de Sevilla, Camino de \\ los Descubrimiento s.n., 41092 Sevilla, Spain (e-mail: \\ rvazquez1@us.es). \\ ** Dept. of Mechanical and Aerospace Engineering, University of \\ California San Diego, La Jolla, CA 92093-0411, USA (e-mail: \\ krstic@ucsd.edu).
}

\begin{abstract}
This paper introduces an explicit full-state boundary feedback law that stabilizes an unstable linear constant-coefficient reaction-diffusion equation on a disk. The backstepping method is used to design the control law. To apply backstepping the system is reduced to an infinite sequence of $1-\mathrm{D}$ systems using Fourier series. $H^{2}$ well-posedness and stability are proved, which implies that the solution is at least continuous on the disk.
\end{abstract}

\section{INTRODUCTION}

In this paper we introduce an explicit full-state boundary feedback law to stabilize an unstable linear constantcoefficient reaction-diffusion equation on a disk-shaped domain. Based on the domain shape we use polar coordinates, and then transform the system into an infinite sequence of 1-D systems using Fourier series, each of which can be independently controlled. For each harmonic we design a feedback law using the backstepping method [8]. The backstepping method has proved itself to be an ubiquitous method for PDE control, with many other applications including, among others, flow control [15, 19], nonlinear PDEs [16], hyperbolic 1-D systems [5, 6, 10], adaptive control [13], wave equations [12], and delays [9].

Backstepping was previously used to design an output feedback law for a convection problem on an annular domain [18], but including the origin makes more difficult the design, as (apparent) singularities appear on the equations and have to be dealt with. Despite this complication, we are able to explicitly find the backstepping kernels and subsequently reconstruct our control law back in physical space, showing that the closed-loop system is well-posed and exponentially stable in the $H^{2}$ norm.

Other design methods could have been applied (see for instace [14] or [3]). Also, there have been specific results on disk- or spherical-shaped domains, such as [17] and [11], however these works assume perfect symmetry of initial conditions which allows to consider only radial variations, thus simplifying the problem considerably.

The structure of the problem is as follows. In Section 2 we introduce the problem and state our main result. We explain our design method and explicitly find the control kernels in Section 3. Next, we prove the stability result in Section 4. We conclude the paper with some remarks in Section 5.

\section{2-D REACTION-DIFFUSION SYSTEM ON A DISK}

Consider the following reaction-diffusion system on a disk, written in polar coordinates $(r, \theta)$ :

$$
u_{t}=\frac{\epsilon}{r}\left(r u_{r}\right)_{r}+\frac{\epsilon}{r^{2}} u_{\theta \theta}+\lambda u,
$$

evolving in the disk $\mathcal{D}_{R}=\{(r, \theta): r \in[0, R], \theta \in[0,2 \pi)\}$, for $t>0$, with boundary conditions

$$
u(t, R, \theta)=U(t, \theta),
$$

where $U(t)$ is the actuation (we assume we can control all the boundary). Note that the system will be unstable for large values of $\lambda$. Denote by $H^{2}\left(\mathcal{D}_{R}\right)$ the space of $H^{2}$ functions on the disk. The following result holds.

Theorem 1. Consider (1)-(2) with initial conditions $u_{0}(x)$ and the following (explicit) full-state feedback law for $U$ :

$$
\begin{aligned}
U(t, \theta)= & \mathcal{F}[u]=-\frac{1}{2 \pi} \frac{\lambda}{\epsilon} \int_{0}^{R} \rho \frac{\mathrm{I}_{1}\left[\sqrt{\frac{\lambda}{\epsilon}\left(R^{2}-\rho^{2}\right)}\right]}{\sqrt{\frac{\lambda}{\epsilon}\left(R^{2}-\rho^{2}\right)}} \\
& \times \int_{-\pi}^{\pi} \frac{\left(R^{2}-\rho^{2}\right) u(t, \rho, \psi)}{R^{2}+\rho^{2}-2 R \rho \cos (\theta-\psi)} d \psi d \rho,
\end{aligned}
$$

where $\mathrm{I}_{1}$ is the first-order modified Bessel function of the first kind, and assume in addition that $u_{0} \in H^{2}\left(\mathcal{D}_{R}\right)$ and verifies the compatibility condition $u_{0}(R)=\mathcal{F}\left[u_{0}\right]$. Then system (1)-(2) has a unique $H^{2}\left(\mathcal{D}_{R}\right)$ solution, and the equilibrium profile $u \equiv 0$ is exponentially stable in the $H^{2}\left(\mathcal{D}_{R}\right)$ norm, i.e., there exists $c_{1}, c_{2}>0$ such that

$$
\|u(t, \cdot)\|_{H^{2}\left(\mathcal{D}_{R}\right)} \leq c_{1} \mathrm{e}^{-c_{2} t}\left\|u_{0}\right\|_{H^{2}\left(\mathcal{D}_{R}\right)} .
$$

In the next sections we sketch the proof of the result. First (Section 3) we explain the rationale behind the method used for reaching (3), and next (Section 4) we show the stability result for the closed loop system.

\section{CONTROL LAW DESIGN}

In this section we explain how the feedback law (3) has been constructed. Given that the angular coordinate is periodic and the system is linear we expand the system by using its Fourier series representation. We stabilize each harmonic independently by using the backstepping method. Finally, we put together all the harmonics reconstructing the feedback law in physical space. 


\subsection{Expansion in Fourier series}

To handle the $\theta$ dependency, we expand both the state $u$ and the control $U$ using a Fourier series:

$$
\begin{aligned}
u(t, r, \theta) & =\sum_{n=-\infty}^{n=\infty} u_{n}(t, r) \mathrm{e}^{i n \theta} \\
U(t, \theta) & =\sum_{n=-\infty}^{n=\infty} U_{n}(t) \mathrm{e}^{i n \theta}
\end{aligned}
$$

for $n \in \mathbb{Z}$, where the coefficients are defined as

$$
\begin{gathered}
u_{n}(t, r)=\frac{1}{2 \pi} \int_{-\pi}^{\pi} u(t, r, \psi) \mathrm{e}^{-i n \psi} d \psi \\
U_{n}(t)=\frac{1}{2 \pi} \int_{-\pi}^{\pi} U(t, \psi) \mathrm{e}^{-i n \psi} d \psi
\end{gathered}
$$

From (1), each coefficient $u_{n}$ verifies the following equation:

$$
u_{n t}=\frac{\epsilon}{r}\left(r u_{n r}\right)_{r}-n^{2} \frac{\epsilon}{r^{2}} u_{n}+\lambda u_{n},
$$

evolving in $r \in[0, R], t>0$, with boundary conditions

$$
u_{n}(t, R)=U_{n}(t)
$$

Thanks to the fact that (1) is linear and the coefficients do not depend on $\theta$, the equations are not coupled and thus we can independently design each $U_{n}$ and later re-assemble all of the harmonics to find $U$.

\subsection{Backstepping transformation}

Our approach to design $U_{n}(t)$ will be to seek a mapping that transforms (9) into the following target system

$$
w_{n t}=\frac{\epsilon}{r}\left(r w_{n r}\right)_{r}-n^{2} \frac{\epsilon}{r^{2}} w_{n},
$$

a stable heat equation (a negative reaction coefficient could also be added if desired), with boundary conditions

$$
w_{n}(t, R)=0
$$

The transformation is defined as follows:

$$
w_{n}(t, r)=u_{n}(t, r)-\int_{0}^{r} K_{n}(r, \rho) u_{n}(t, \rho) d \rho,
$$

and then $U_{n}(t)$ will be found from (13) at $r=R$.

To find the kernel equations we proceed, as usual in the backstepping method [8], by substituting both the original and target systems in the transformation and integrating by parts when possible. We skip the details for lack of space. We obtain the following PDE that the kernel must verify:

$$
\frac{\left(r K_{n r}\right)_{r}}{r}-\left(\rho\left(\frac{K_{n}}{\rho}\right)_{\rho}\right)_{\rho}-n^{2}\left(\frac{1}{r^{2}}-\frac{1}{\rho^{2}}\right) K_{n}=\frac{\lambda}{\epsilon} K_{n} .
$$

Also, the following boundary conditions have to be verified

$$
\begin{aligned}
& 0=\lambda+2 \epsilon \frac{d}{d r}\left(K_{n}(r, r)\right), \\
& 0=K_{n}(r, 0), \\
& 0=\lim _{\rho \rightarrow 0}\left(\left(\frac{K_{n}(r, \rho)}{\rho}\right)_{\rho} \rho\right) .
\end{aligned}
$$

Boundary condition (15) integrates to

$$
K_{n}(r, r)=-\int_{0}^{r} \frac{\lambda}{2 \epsilon} d \rho=-\frac{\lambda r}{2 \epsilon},
$$

where we have used (15) at $r=0$ (i.e., $K_{n}(0,0)=0$ ). Finally (15) can be written as

$$
0=K_{n \rho}(r, 0)-\lim _{\rho \rightarrow 0} \frac{K_{n}(r, \rho)}{\rho} .
$$

However, from the second boundary condition $K_{n}(r, 0)=$ 0 . Assuming $K_{n}(r, \rho)$ is differentiable in $\rho$, then one has $K_{n \rho}(r, 0)=\lim _{\rho \rightarrow 0} \frac{K_{n}(r, \rho)}{\rho}$ and (17) is automatically verified. Thus the only boundary conditions that have to be considered are (18) and (16).

\subsection{Explicitly solving the kernel equations}

To solve (14) with boundary conditions (18) and (16), let us make the change $K(r, \rho)=G(r, \rho) \rho\left(\frac{\rho}{r}\right)^{|n|}$. We end up with

$$
G_{n r r}+(1-2|n|) \frac{G_{n r}}{r}-G_{n \rho \rho}-(1+2|n|) \frac{G_{n \rho}}{\rho}=\frac{\lambda}{\epsilon} G_{n},
$$

with only one boundary condition (since (16) is automatically verified):

$$
G_{n}(r, r)=-\frac{\lambda}{2 \epsilon}
$$

Now assume a solution $G_{n}(r, \rho)$ of the form $G_{n}(r, \rho)=$ $\Phi\left(\left(\frac{\lambda}{\epsilon}\left(r^{2}-\rho^{2}\right)\right)^{1 / 2}\right)$. Notice it is independent of $n$. Expressing the derivatives of $G_{n}$ in terms of $\Phi$ and replacing them in (20) we find

$$
\frac{\lambda}{\epsilon} \Phi^{\prime \prime}+3 \frac{\lambda}{\epsilon}\left(\frac{\lambda}{\epsilon}\left(r^{2}-\rho^{2}\right)\right)^{-1 / 2} \Phi^{\prime}=\frac{\lambda}{\epsilon} \Phi
$$

with boundary condition $\Phi(0)=-\frac{\lambda}{2 \epsilon}$. Denoting $x=$ $\left(\frac{\lambda}{\epsilon}\left(r^{2}-\rho^{2}\right)\right)^{1 / 2}$ and the derivatives with respect to $x$ with a dot, we can write (22) as

$$
\ddot{\Phi}(x)+\frac{3}{x} \dot{\Phi}(x)-\Phi(x)=0,
$$

and finally calling $\Psi(x)=x \Phi(x)$, we obtain

$$
\left(\frac{\ddot{\Psi}}{x}-2 \frac{\dot{\Psi}}{x^{2}}+2 \frac{\Psi}{x^{3}}\right)+\frac{3}{x}\left(\frac{\dot{\Psi}}{x}-\frac{\Psi}{x^{2}}\right)-\frac{\Psi}{x}=0,
$$

which cross-multiplied by $x^{3}$ gives

$$
x^{2} \ddot{\Psi}+x \dot{\Psi}-\left(1+x^{2}\right) \Psi=0,
$$

which is Bessel's modified differential equation of order 1 (see [1], p. 374, Sec. 9.6.1). The (bounded) solution is

$$
\Psi(x)=C_{1} \mathrm{I}_{1}(x),
$$


and going backwards we obtain

$$
\Phi(x)=C_{1} \frac{\mathrm{I}_{1}(x)}{x} .
$$

Noticing that $\lim _{x \rightarrow 0} \frac{\mathrm{I}_{1}(x)}{x}=1 / 2$, we get $C_{1}=-\frac{\lambda}{\epsilon}$ (from the boundary condition at $x=0$ ). Therefore, we obtain, by undoing the change of variables to recover $G_{n}$,

$$
G_{n}(r, \rho)=-\frac{\lambda}{\epsilon} \frac{\mathrm{I}_{1}\left[\sqrt{\frac{\lambda}{\epsilon}\left(r^{2}-\rho^{2}\right)}\right]}{\sqrt{\frac{\lambda}{\epsilon}\left(r^{2}-\rho^{2}\right)}},
$$

and therefore

$$
K_{n}(r, \rho)=-\rho\left(\frac{\rho}{r}\right)^{|n|} \frac{\lambda}{\epsilon} \frac{\mathrm{I}_{1}\left[\sqrt{\frac{\lambda}{\epsilon}\left(r^{2}-\rho^{2}\right)}\right]}{\sqrt{\frac{\lambda}{\epsilon}\left(r^{2}-\rho^{2}\right)}} .
$$

\subsection{Finding the 2-D backstepping kernel}

The backstepping transformation, written in real space, is $w(t, r, \theta)=u(t, r, \theta)-\int_{0}^{r} \int_{-\pi}^{\pi} K(r, \rho, \theta, \psi) u(t, \rho, \psi) d \psi d \rho$,

where $K$ is recovered from its harmonics $K_{n}$ as

$$
K(r, \rho, \theta, \psi)=\sum_{n=-\infty}^{n=\infty} \frac{K_{n}(r, \rho)}{2 \pi} \mathrm{e}^{n i(\theta-\psi)} .
$$

Using (14), we compute $K$ as

$$
K=-\rho \frac{\lambda}{2 \pi \epsilon} \frac{\mathrm{I}_{1}\left[\sqrt{\frac{\lambda}{\epsilon}\left(r^{2}-\rho^{2}\right)}\right]}{\sqrt{\frac{\lambda}{\epsilon}\left(r^{2}-\rho^{2}\right)}} \sum_{n=-\infty}^{n=\infty}\left(\frac{\rho}{r}\right)^{|n|} \mathrm{e}^{n i(\theta-\psi)}
$$

and since $\rho \leq r$, this can be summed (except at $\rho=r$ )

$$
\begin{aligned}
& \sum_{n=-\infty}^{n=\infty}\left(\frac{\rho}{r}\right)^{|n|} \mathrm{e}^{n i(\theta-\psi)} \\
= & \sum_{n=0}^{n=\infty}\left(\frac{\rho}{r}\right)^{n} \mathrm{e}^{n i(\theta-\psi)}+\sum_{n=-\infty}^{n=0}\left(\frac{\rho}{r}\right)^{-n} \mathrm{e}^{n i(\theta-\psi)}-1 \\
= & \sum_{n=0}^{n=\infty}\left(\frac{\rho}{r} \mathrm{e}^{i(\theta-\psi)}\right)^{n}+\sum_{n=0}^{n=\infty}\left(\frac{\rho}{r} \mathrm{e}^{-i(\theta-\psi)}\right)^{n}-1 \\
= & \frac{1}{1-\left(\frac{\rho}{r} \mathrm{e}^{i(\theta-\psi)}\right)}+\frac{1}{1-\left(\frac{\rho}{r} \mathrm{e}^{-i(\theta-\psi)}\right)}-1 \\
= & \frac{1-\frac{\rho^{2}}{r^{2}}}{1+\frac{\rho^{2}}{r^{2}}-2 \frac{\rho}{r} \cos (\theta-\psi)}
\end{aligned}
$$

which is the Poisson kernel (see [7],p.41, a function that tends to a Dirac delta $\delta(\theta-\psi)$ when $r$ goes to $\rho)$. Thus the function (by itself) is unbounded at $r=\rho$ but valid as an integral kernel; finally

$$
\begin{aligned}
K(r, \rho, \theta, \psi)= & -\frac{\lambda \rho}{2 \pi \epsilon} \frac{\mathrm{I}_{1}\left[\sqrt{\frac{\lambda}{\epsilon}\left(r^{2}-\rho^{2}\right)}\right]}{\sqrt{\frac{\lambda}{\epsilon}\left(r^{2}-\rho^{2}\right)}} \\
& \times \frac{r^{2}-\rho^{2}}{r^{2}+\rho^{2}-2 r \rho \cos (\theta-\psi)},
\end{aligned}
$$

and the control law (3) is found by setting $r=R$ in (30).

\section{PROOF OF CLOSED-LOOP STABILITY}

\subsection{Definition of Sobolev norms}

To proceed with the proof of closed-loop stability, we first need to define the appropriate norms. Since all the norms will be defined on the disk of radius $R$ we will omit the symbol $\mathcal{D}_{R}$ in the norm. Define the $L^{2}$ norm in the disk as

$$
\|f\|_{L^{2}}=\left(\int_{0}^{R} \int_{-\pi}^{\pi}|f|^{2}(r, \theta) r d \theta d r\right)^{1 / 2} .
$$

The Sobolev norm $\|\cdot\|_{H^{2}}$ is defined as [2]

$$
\|f\|_{H^{2}}=\|f\|_{L^{2}}+\left\|\frac{\partial^{2} f}{\partial x^{2}}\right\|_{L^{2}}+\left\|\frac{\partial^{2} f}{\partial y^{2}}\right\|_{L^{2}}+2\left\|\frac{\partial^{2} f}{\partial x \partial y}\right\|_{L^{2}},
$$

however we will compute the derivatives in the $H^{2}$ norm in polar coordinates, as follows

$$
\frac{\partial f}{\partial x}=\cos \theta \frac{\partial f}{\partial r}-\frac{\sin \theta}{r} \frac{\partial f}{\partial \theta}, \frac{\partial f}{\partial y}=\sin \theta \frac{\partial f}{\partial r}+\frac{\cos \theta}{r} \frac{\partial f}{\partial \theta} .
$$

\subsection{Stability of the target system}

To prove well-possedness and stability of (1)-(2) with feedback law (3), we first remark that the closed-loop is equivalent (by using transformation (30)) to the target system (11)-(12), which written back in physical space is

$$
\begin{aligned}
w_{t} & =\frac{\epsilon}{r}\left(r w_{r}\right)_{r}+\frac{\epsilon}{r^{2}} w_{\theta \theta}, \\
w(t, R, \theta) & =0 .
\end{aligned}
$$

We next state a result about (38)-(39).

Proposition 2. Consider the system (38)-(39) with initial conditions $w_{0}(r, \theta)$. Then, if $w_{0} \in H^{2}$ and $w_{0}(R, \theta)=0$, then $w \in \mathcal{C}\left[[0, \infty), H^{2}\right]$ and also

$$
\|w(t, \cdot)\|_{H^{2}} \leq D \mathrm{e}^{-\alpha t}\left\|w_{0}\right\|_{H^{2}} .
$$

where $D$ and $\alpha$ are positive constants.

The well-posedness part of the result is standard (see for instance [4], pages 326 and 328). The stability estimate is easy to obtain using a Lyapunov argument, which we skip due to lack of space.

\subsection{Inverse transformation}

Using standard arguments (see for instance [19]) one can show that (30) is invertible. It is also possible to show that if we write the inverse as

$$
u(t, r, \theta)=w(t, r, \theta)+\int_{0}^{r} \int_{-\pi}^{\pi} L(r, \rho, \theta, \psi) w(t, \rho, \psi) d \psi d \rho,
$$

then, breaking down the kernel $L$ in its Fourier components $L_{n}$ and computing the partial differential equation verified by each $L_{n}$, one finds that it has a very similar structure to the $K_{n}$ kernel equations, and solving it, $L$ is thus found to have a very similar structure to (34), as follows

$$
\begin{aligned}
L(r, \rho, \theta, \psi)= & -\frac{\lambda \rho}{2 \pi \epsilon} \frac{\mathrm{J}_{1}\left[\sqrt{\frac{\lambda}{\epsilon}\left(r^{2}-\rho^{2}\right)}\right]}{\sqrt{\frac{\lambda}{\epsilon}\left(r^{2}-\rho^{2}\right)}} \\
& \times \frac{r^{2}-\rho^{2}}{r^{2}+\rho^{2}-2 r \rho \cos (\theta-\psi)},
\end{aligned}
$$

where $\mathrm{J}_{1}$ is the first order Bessel function of the first kind. 


\subsection{Proof of Theorem 1}

Since the transformation is invertible, and the compatibility condition $w_{0}(R, \theta)=0$ is translated in the original system to the condition $u_{0}(R, \theta)=\mathcal{F}[u]$, Proposition 2 can be applied to directly prove Theorem 1 , if we can show that both the direct and inverse transformation transform $H^{2}$ functions back into $H^{2}$ functions. The next proposition gives this result, applied to the functional structure shared by both transformations.

Proposition 3. Assume that the function $g(r, \theta)$ is related to the function $f(r, \theta)$ by means of the transformation

$g(r, \theta)=f(r, \theta)+\int_{0}^{r} \int_{-\pi}^{\pi} \rho F(r, \rho) P(r, \rho, \theta-\psi) f(\rho, \psi) d \psi d \rho$,

where $F(r, \rho) \in \mathcal{C}^{2}(\mathcal{T})$, where $\mathcal{T}=\{(r, \rho): 0 \leq \rho \leq r \leq$ $R\}$ and where $P(r, \rho, \theta-\psi)$ is the Poisson kernel, i.e.,

$$
P(r, \rho, \theta-\psi)=\frac{1}{2 \pi} \frac{r^{2}-\rho^{2}}{r^{2}+\rho^{2}-2 r \rho \cos (\theta-\psi)} .
$$

Then:

$$
\|g\|_{L^{2}} \leq C_{0}\|f\|_{L^{2}},\|g\|_{H^{2}} \leq C_{2}\|f\|_{H^{2}}
$$

where the constants $C_{i}$ depend only on $R$ and $F(r, \rho)$.

The use of $F(r, \rho)$ in Proposition 3 allows to apply it to both the direct and inverse transformation (which would have a similar, but different, $F$, containing the Bessel functions $I_{1}$ and $J_{1}$, respectively). To prove the proposition, we state and prove a number of technical lemmas. First we take care of the $L^{2}$ norm.

\section{Lemma 4 .}

$$
\int_{-\pi}^{\pi}\left|\int_{-\pi}^{\pi} P(r, \rho, \theta-\psi) f(\rho, \psi) d \psi\right|^{2} d \theta \leq \int_{-\pi}^{\pi}|f(\rho, \theta)|^{2} d \theta
$$

and also, defining $H\left(n_{1}, n_{2}, \phi\right)=\cos ^{n_{1}}(\phi) \sin ^{n_{2}}(\phi)$ for positive $n_{1}, n_{2}$, we have

$$
\begin{aligned}
& \int_{-\pi}^{\pi}\left|\int_{-\pi}^{\pi} P(r, \rho, \theta-\psi) H\left(n_{1}, n_{2}, \theta-\psi\right) f(\rho, \psi) d \psi\right|^{2} d \theta \\
\leq & \int_{-\pi}^{\pi}|f(\rho, \theta)|^{2} d \theta .
\end{aligned}
$$

Proof Using Fourier series, if $f_{n}(\rho)$ are the coefficients of $f(\rho, \theta)$ and noting that

$$
P(r, \rho, \theta)=\frac{1}{2 \pi} \sum_{n=-\infty}^{n=\infty}\left(\frac{\rho}{r}\right)^{|n|} \mathrm{e}^{i n \theta},
$$

then,

$$
\begin{aligned}
& \int_{-\pi}^{\pi} P(r, \rho, \theta-\psi) f(\rho, \psi) d \psi \\
= & \frac{1}{2 \pi} \sum_{n=-\infty}^{n=\infty} \sum_{m=-\infty}^{m=\infty}\left(\frac{\rho}{r}\right)^{|n|} f_{m}(\rho) \int_{-\pi}^{\pi} \mathrm{e}^{i n(\theta-\psi)} \mathrm{e}^{i m \psi} d \psi \\
= & \sum_{n=-\infty}^{n=\infty}\left(\frac{\rho}{r}\right)^{|n|} f_{n}(\rho) \mathrm{e}^{i n \theta}
\end{aligned}
$$

Here, we have used the orthogonality property of Fourier series (the same conclusion can be reached using the convolution theorem). Now, by Parseval's theorem

$$
\begin{aligned}
& \int_{-\pi}^{\pi}\left|\int_{-\pi}^{\pi} P(r, \rho, \theta-\psi) f(\rho, \psi) d \psi\right|^{2} d \theta \\
= & 2 \pi \sum_{n=-\infty}^{n=\infty}\left|\left(\frac{\rho}{r}\right)^{|n|} f_{n}(\rho)\right|^{2} \leq 2 \pi \sum_{n=-\infty}^{n=\infty}\left|f_{n}(\rho)\right|^{2} \\
= & \int_{-\pi}^{\pi}|f(\rho, \theta)|^{2} d \theta .
\end{aligned}
$$

To obtain the result for (47) we can mimic the proof but we would need to show that the Fourier coefficients of $P(r, \rho, \theta) \cos ^{n_{1}}(\theta) \sin ^{n_{2}}(\theta)$ are less or equal than one. We will just show it by induction on $n_{1}$ for $n_{2}=0$, since the proof would be the same for $n_{2}$. For $n_{1}=0$ it is obvious. Assuming that the Fourier coefficients of $P(r, \rho, \theta) \cos ^{n_{1}}(\theta)$, denoted as $a_{n}$, are such that $\left|a_{n}\right| \leq 1$, we have for $n_{1}+1$ that

$$
\begin{aligned}
P(r, \rho, \theta) \cos ^{n_{1}+1}(\theta) & =\sum_{n=-\infty}^{n=\infty} a_{n} \mathrm{e}^{i n \theta} \cos \theta \\
& =\sum_{n=-\infty}^{n=\infty} a_{n} \mathrm{e}^{i n \theta} \frac{\mathrm{e}^{i \theta}+\mathrm{e}^{-i \theta}}{2} \\
& =\sum_{n=-\infty}^{n=\infty} \frac{a_{n-1}}{2} \mathrm{e}^{i n \theta}+\sum_{n=-\infty}^{n=\infty} \frac{a_{n+1}}{2} \mathrm{e}^{i n \theta} \\
& =\sum_{n=-\infty}^{n=\infty} \frac{a_{n-1}+a_{n+1}}{2} \mathrm{e}^{i n \theta},
\end{aligned}
$$

and obviously the new coefficients $b_{n}=\frac{a_{n-1}+a_{n+1}}{2}$ verify $\left|b_{n}\right| \leq 1$. Thus the result follows.

Lemma 5. If

$g(r, \theta)=f(r, \theta)+\int_{0}^{r} \int_{-\pi}^{\pi} \rho F(r, \rho) P(r, \rho, \theta-\psi) f(\rho, \psi) d \psi d \rho$,

where $F(r, \rho) \in \mathcal{C}(\mathcal{T})$, then

$$
\|g\|_{L^{2}} \leq C_{0}\|f\|_{L^{2}}
$$

Proof First, since $F(r, \rho) \in \mathcal{C}(\mathcal{T})$, we can bound $F$ :

$$
\forall(r, \rho) \in \mathcal{T} \quad|F(r, \rho)| \leq M
$$

In the proof we skip the function dependencies for lack of space. We find

$$
\begin{aligned}
\|g\|_{L^{2}}^{2} & =\int_{0}^{R} \int_{-\pi}^{\pi}\left|f+\int_{0}^{r} \int_{-\pi}^{\pi} F P f d \psi \rho d \rho\right|^{2} r d \theta d r \\
& \leq 2\|f\|_{L^{2}}^{2}+M^{2} \int_{0}^{R} r^{3} \int_{0}^{r} \int_{-\pi}^{\pi}\left|\int_{-\pi}^{\pi} P f d \psi\right|^{2} d \theta \rho d \rho d r \\
& \leq 2\|f\|_{L^{2}}^{2}+M^{2} \int_{0}^{R} r^{3} \int_{0}^{r} \int_{-\pi}^{\pi}|f(\rho, \theta)|^{2} \rho d \theta d \rho d r \\
& \leq 2\left(1+\frac{M^{2} R^{4}}{8}\right)\|f\|_{L^{2}}^{2}
\end{aligned}
$$

thus by setting $C_{0}=2\left(1+\frac{M^{2} R^{4}}{8}\right)$ we have proven the lemma.

Thus we obtain the $L^{2}$ bound of Proposition 3 . To compute higher norms we use the next lemma.

Lemma 6. Call $\hat{P}(r, \rho, \theta-\psi)=P(r, \rho, \theta-\psi)\left(\frac{\rho}{r}\right)^{n_{1}} \cos (\theta-$ $\psi)^{n_{2}} \sin (\theta-\psi)^{n_{3}}$ for any integer $n_{1}, n_{2}, n_{3} \geq 0$. Then, if $g(r, \theta)=\int_{0}^{r} \int_{-\pi}^{\pi} \hat{F}(r, \rho, \theta) \hat{P}(r, \rho, \theta-\psi) f(\rho, \psi) d \psi d \rho$, 
where $\hat{F}(r, \rho, \theta) \in \mathcal{C}^{1}(\mathcal{T}) \times \mathcal{C}^{1}([-\pi, \pi])$ and $\hat{F}(r, 0, \theta)=0$, then

$$
\begin{aligned}
g_{x}= & \int_{0}^{r} \int_{-\pi}^{\pi}\left(\frac{r \hat{F}_{r}+\rho \hat{F}_{\rho}+\hat{F}}{r}\right) \hat{P} \cos \theta f(\rho, \psi) d \psi d \rho \\
& -\int_{0}^{r} \int_{-\pi}^{\pi} \frac{1}{r} \frac{\partial \hat{F}(r, \rho, \theta)}{\partial \theta} \hat{P}(r, \rho, \theta-\psi) \sin \theta f(\rho, \psi) d \psi d \rho \\
& +\int_{0}^{r} \int_{-\pi}^{\pi} \hat{F} \frac{\rho}{r} \hat{P}(r, \rho, \theta-\psi)\left[\cos (\theta-\psi) f_{x}(\rho, \psi)\right. \\
& \left.-\sin (\theta-\psi) f_{y}(\rho, \psi)\right] d \psi d \rho
\end{aligned}
$$

and

$$
\begin{aligned}
g_{y}= & \int_{0}^{r} \int_{-\pi}^{\pi}\left(\frac{r \hat{F}_{r}+\rho \hat{F}_{\rho}+\hat{F}}{r}\right) \hat{P} \sin \theta f(\rho, \psi) d \psi d \rho \\
& +\int_{0}^{r} \int_{-\pi}^{\pi} \frac{1}{r} \frac{\partial \hat{F}(r, \rho, \theta)}{\partial \theta} \hat{P}(r, \rho, \theta-\psi) \cos \theta f(\rho, \psi) d \psi d \rho \\
& +\int_{0}^{r} \int_{-\pi}^{\pi} \hat{F} \frac{\rho}{r} \hat{P}(r, \rho, \theta-\psi)\left[\cos (\theta-\psi) f_{y}(\rho, \psi)\right. \\
& \left.+\sin (\theta-\psi) f_{x}(\rho, \psi)\right] d \psi d \rho .
\end{aligned}
$$

Proof By direct differentiation, we find that

$$
\begin{aligned}
g_{x}= & \cos \theta \frac{\partial g}{\partial r}-\frac{\sin \theta}{r} \frac{\partial g}{\partial \theta} \\
= & \cos \theta \hat{F}(r, r, \theta) f(r, \theta) \chi\left(n_{3}\right) \\
& +\int_{0}^{r} \int_{-\pi}^{\pi} \hat{F}_{r}(r, \rho, \theta) \cos \theta \hat{P}(r, \rho, \theta-\psi) f(\rho, \psi) d \psi d \rho \\
& -\int_{0}^{r} \int_{-\pi}^{\pi} \frac{1}{r} \frac{\partial \hat{F}(r, \rho, \theta)}{\partial \theta} \hat{P}(r, \rho, \theta-\psi) \sin \theta f(\rho, \psi) d \psi d \rho \\
& +\int_{0}^{r} \int_{-\pi}^{\pi} \hat{F}(r, \rho, \theta) \cos \theta \frac{\partial}{\partial r} \hat{P}(r, \rho, \theta-\psi) f(\rho, \psi) d \psi d \rho \\
& -\int_{0}^{r} \int_{-\pi}^{\pi} \hat{F}(r, \rho, \theta) \frac{\sin \theta}{r} \frac{\partial}{\partial \theta} \hat{P}(r, \rho, \theta-\psi) f(\rho, \psi) d \psi d \rho,
\end{aligned}
$$

where $\chi\left(n_{3}\right)=1$ if $n_{3}=0$, and zero otherwise. Noticing that $\hat{P}(r, \rho, \theta-\psi)=\Phi\left(\frac{\rho}{r}, \theta-\psi\right)$, we can see by the chain rule that

$$
\begin{aligned}
\frac{\partial}{\partial r} \hat{P}(r, \rho, \theta-\psi) & =-\frac{\rho}{r^{2}} \Phi_{1}\left(\frac{\rho}{r}, \theta-\psi\right), \\
\frac{\partial}{\partial \rho} \hat{P}(r, \rho, \theta-\psi) & =\frac{1}{r} \Phi_{1}\left(\frac{\rho}{r}, \theta-\psi\right),
\end{aligned}
$$

where $\Phi_{1}$ is the derivative of $\Phi$ with respect to its first argument, and therefore we can derive the identity

$$
\frac{\partial}{\partial r} \hat{P}(r, \rho, \theta-\psi)=-\frac{\rho}{r} \frac{\partial}{\partial \rho} \hat{P}(r, \rho, \theta-\psi) .
$$

Similarly,

$$
\frac{\partial}{\partial \theta} \hat{P}(r, \rho, \theta-\psi)=-\frac{\partial}{\partial \psi} \hat{P}(r, \rho, \theta-\psi) .
$$

Thus, if we integrate by parts in the last two lines of (59) and expand $\cos \theta=\cos (\theta-\psi+\psi)=\cos (\theta-\psi) \cos (\psi)-$ $\sin (\theta-\psi) \sin \psi$ and $\sin \theta=\sin (\theta-\psi+\psi)=\sin (\theta-$ $\psi) \cos (\psi)+\cos (\theta-\psi) \sin \psi$, we reach the result. The proof for $g_{y}$ is carried exactly in the same fashion.

Applying the previous lemma we can obtain expressions for $g_{x}, g_{y}, g_{x x}, g_{x y}$ and $g_{y y}$. Due to lack of space we only show $g_{x}$ and $g_{x x}$ (other derivatives are similar).
Lemma \%. If

$g(r, \theta)=f(r, \theta)+\int_{0}^{r} \int_{-\pi}^{\pi} \rho F(r, \rho) P(r, \rho, \theta-\psi) f(\rho, \psi) d \psi d \rho$,

where $F(r, \rho) \in \mathcal{C}^{1}(\mathcal{T})$, then

$$
\begin{aligned}
g_{x}= & f_{x}+\int_{0}^{r} \int_{-\pi}^{\pi}\left(2 F(r, \rho) \frac{\rho}{r}+\rho F_{\rho}(r, \rho) \frac{\rho}{r}+\rho F_{r}(r, \rho)\right) \\
& \times P(r, \rho, \theta-\psi) \cos \theta f(\rho, \psi) d \psi d \rho \\
& +\int_{0}^{r} \int_{-\pi}^{\pi} \rho F(r, \rho) \frac{\rho}{r} P(r, \rho, \theta-\psi)\left[\cos (\theta-\psi) f_{x}(\rho, \psi)\right. \\
& \left.-\sin (\theta-\psi) f_{y}(\rho, \psi)\right] d \psi d \rho .
\end{aligned}
$$

Using these expressions we can bound the first derivatives: Lemma 8. If

$g(r, \theta)=f(r, \theta)+\int_{0}^{r} \int_{-\pi}^{\pi} \rho F(r, \rho) P(r, \rho, \theta-\psi) f(\rho, \psi) d \psi d \rho$,

where $F(r, \rho) \in \mathcal{C}^{1}(\mathcal{T})$, then

$$
\begin{gathered}
\left\|g_{x}\right\|_{L^{2}} \leq C_{3}\|f\|_{L^{2}}+C_{4}\left(\left\|f_{x}\right\|_{L^{2}}+\left\|f_{y}\right\|_{L^{2}}\right), \\
\left\|g_{y}\right\|_{L^{2}} \leq C_{3}\|f\|_{L^{2}}+C_{4}\left(\left\|f_{x}\right\|_{L^{2}}+\left\|f_{y}\right\|_{L^{2}}\right) .
\end{gathered}
$$

Proof First, since $F(r, \rho) \in \mathcal{C}^{1}(\mathcal{T})$ :

$$
\forall(r, \rho) \in \mathcal{T} \quad\left|F_{r}(r, \rho)\right| \leq M_{r}, \quad\left|F_{\rho}(r, \rho)\right| \leq M_{\rho}
$$

Then

$$
\begin{aligned}
\left|g_{x}\right|^{2} \leq & 4\left|f_{x}\right|^{2}+4 \mid \cos \theta \int_{0}^{r}\left(2 F \frac{\rho}{r}+\rho F_{\rho} \frac{\rho}{r}+\rho F_{r}\right) \\
& \times\left.\int_{-\pi}^{\pi} P f d \psi d \rho\right|^{2} \\
& +4\left|\int_{0}^{r} \rho F \frac{\rho}{r} \int_{-\pi}^{\pi} P \cos (\theta-\psi) f_{x} d \psi d \rho\right|^{2} \\
& +4\left|\int_{0}^{r} \rho F \frac{\rho}{r} \int_{-\pi}^{\pi} P \sin (\theta-\psi) f_{y} d \psi d \rho\right|^{2} \\
\leq & 4\left|f_{x}\right|^{2}+12\left(2 M^{2}+\frac{r^{2}}{4} M_{\rho}^{2}+\frac{r^{2}}{2} M_{r}^{2}\right) \\
& \times \int_{0}^{r}\left|\int_{-\pi}^{\pi} P f d \psi\right|^{2} \rho d \rho \\
& +r^{2} M^{2} \int_{0}^{r}\left|\int_{-\pi}^{\pi} P \cos (\theta-\psi) f_{x} d \psi\right|^{2} \rho d \rho \\
& +r^{2} M^{2} \int_{0}^{r}\left|\int_{-\pi}^{\pi} P \sin (\theta-\psi) f_{y} d \psi\right|^{2} \rho d \rho .
\end{aligned}
$$

Thus,

$$
\begin{aligned}
\left\|g_{x}\right\|_{L^{2}}^{2}= & 4\left\|f_{x}\right\|_{L^{2}}^{2}+3\left(M^{2} R^{2}+\frac{R^{4}}{4} M_{\rho}^{2}+\frac{R^{4}}{2} M_{r}^{2}\right)\|f\|_{L^{2}}^{2} \\
& +\frac{R^{4}}{4} M^{2}\left(\left\|f_{x}\right\|_{L^{2}}^{2}+\left\|f_{y}\right\|_{L^{2}}^{2}\right) .
\end{aligned}
$$

Proceeding similarly for $g_{y}$ we prove the lemma.

To obtain $H^{2}$ norm bounds we only two additional results. In the first one we only show the expression of $g_{x x}\left(g_{x y}\right.$ and $g_{y y}$ share a similar functional structure). 
Lemma 9. If

$g(r, \theta)=f(r, \theta)+\int_{0}^{r} \int_{-\pi}^{\pi} \rho F(r, \rho) P(r, \rho, \theta-\psi) f(\rho, \psi) d \psi d \rho$,

where $F(r, \rho) \in \mathcal{C}^{2}(\mathcal{T})$, then

$$
\begin{aligned}
g_{x x}= & f_{x x}+\int_{0}^{r} \int_{-\pi}^{\pi}\left[H_{2} \cos ^{2} \theta+\frac{H_{1}}{r} \sin ^{2} \theta\right] P f d \psi d \rho \\
& +\int_{0}^{r} \int_{-\pi}^{\pi} 2 H_{1} \frac{\rho}{r} P \cos (\theta)\left[\cos (\theta-\psi) f_{x}-\sin (\theta-\psi)\right. \\
& \left.\times f_{y}\right] d \psi d \rho+\int_{0}^{r} \int_{-\pi}^{\pi} \rho F \frac{\rho^{2}}{r^{2}} P\left[\cos ^{2}(\theta-\psi) f_{x x}\right. \\
& \left.-2 \sin (\theta-\psi) \cos (\theta-\psi) f_{x y}+\sin ^{2}(\theta-\psi) f_{y y}\right] d \psi d \rho,
\end{aligned}
$$

where

$$
\begin{aligned}
& H_{1}(r, \rho)=2 F(r, \rho) \frac{\rho}{r}+\rho F_{\rho}(r, \rho) \frac{\rho}{r}+\rho F_{r}(r, \rho), \\
& H_{2}(r, \rho)=\left(\partial_{r}+\frac{\rho}{r} \partial_{\rho}+\frac{1}{r}\right) H_{1}(r, \rho) .
\end{aligned}
$$

Proof It is proven by applying Lemma 6 to Lemma 7.

Next lemma bounds the norm of the second derivatives. Lemma 10. If

$g(r, \theta)=f(r, \theta)+\int_{0}^{r} \int_{-\pi}^{\pi} \rho F(r, \rho) P(r, \rho, \theta-\psi) f(\rho, \psi) d \psi d \rho$,

where $F(r, \rho) \in \mathcal{C}^{2}(\mathcal{T})$, then

$$
\begin{aligned}
& \left\|g_{x x}\right\|_{L^{2}} \leq C_{5}\|f\|_{H^{1}}+C_{6}\left(\left\|f_{x x}\right\|_{L^{2}}+\left\|f_{x y}\right\|_{L^{2}}+\left\|f_{y y}\right\|_{L^{2}}\right), \\
& \left\|g_{x y}\right\|_{L^{2}} \leq C_{5}\|f\|_{H^{1}}+C_{6}\left(\left\|f_{x x}\right\|_{L^{2}}+\left\|f_{x y}\right\|_{L^{2}}+\left\|f_{y y}\right\|_{L^{2}}\right), \\
& \left\|g_{y y}\right\|_{L^{2}} \leq C_{5}\|f\|_{H^{1}}+C_{6}\left(\left\|f_{x x}\right\|_{L^{2}}+\left\|f_{x y}\right\|_{L^{2}}+\left\|f_{y y}\right\|_{L^{2}}\right) .
\end{aligned}
$$

Proof The proof is carried out as the proof of Lemma 8. The only difference is the integral with the term $\frac{\rho}{r^{2}}$ which has to be treated with care to avoid a singularity,as follows.

$$
\begin{aligned}
& \int_{0}^{R} \int_{-\pi}^{\pi}\left|\int_{0}^{r} \frac{\rho}{r^{2}} \int_{-\pi}^{\pi} P f d \psi d \rho\right|^{2} d \theta r d r \\
= & \int_{0}^{R} \int_{-\pi}^{\pi}\left|\int_{0}^{r} \frac{\rho}{r^{3 / 2}} \int_{-\pi}^{\pi} P f d \psi d \rho\right|^{2} d \theta d r \\
\leq & \int_{0}^{R} \frac{1}{r^{2}} \int_{0}^{r} \rho^{2} \int_{-\pi}^{\pi}\left|\int_{-\pi}^{\pi} P f d \psi\right|^{2} d \theta d \rho d r \\
\leq & \int_{0}^{R} \rho^{2} \int_{\rho}^{R} \frac{1}{r^{2}} \int_{-\pi}^{\pi}|f|^{2} d \theta d r d \rho \\
= & \int_{0}^{R} \rho^{2}\left(\frac{1}{\rho}-\frac{1}{R}\right) \int_{-\pi}^{\pi}|f|^{2} d \theta d \rho \\
\leq & \int_{0}^{R} \int_{-\pi}^{\pi}|f|^{2} d \theta \rho d \rho .
\end{aligned}
$$

Proceeding similarly for $g_{y y}$ and $g_{x y}$, whose expressions are very similar to $g_{x x}$, we prove the lemma.

Thus we have proved the last part of Proposition 3.

\section{CONCLUSION}

We have shown an explicit design to stabilize a constantcoefficient reaction-diffusion equation on a disk using boundary control. The resulting control law uses full state feedback, but following similar ideas it is possible to design an observer and an output-feedback law. The method can be extended to 3 (or higher) dimensions by using spherical harmonics, obtaining similar results in terms of Bessel functions and Poisson kernels, however higher Sobolev spaces are needed to obtain continuity of solutions.

\section{REFERENCES}

[1] M. Abramowitz and I. A. Stegun. Handbook of mathematical functions. 9th Edition, Dover, 1965.

[2] R. A. Adams and J. Fournier. Sobolev Spaces. Academic Press, 2003.

[3] V. Barbu. Boundary Stabilization of Equilibrium Solutions to Parabolic Equations. IEEE Transactions on Automatic Control, 58:2416-2420, 2013.

[4] H. Brezis. Functional analysis, Sobolev spaces and Partial Differential Equations. Springer, 2011.

[5] J.-M. Coron, R. Vazquez, M. Krstic, and G. Bastin. Local Exponential $H^{2}$ Stabilization of a $2 \times 2$ Quasilinear Hyperbolic System using Backstepping. SIAM J. Control Optim., 51:2005-2035, 2013.

[6] F. Di Meglio, R. Vazquez, and M. Krstic. Stabilization of a system of $n+1$ coupled first-order hyperbolic linear PDEs with a single boundary input. IEEE Transactions on Automatic Control, PP, 2013.

[7] L.C. Evans. Partial Differential Equations. AMS, Providence, Rhode Island, 1998.

[8] M. Krstic and A. Smyshlyaev. Boundary Control of PDEs. SIAM, 2008

[9] M. Krstic. Delay Compensation for Nonlinear, Adaptive, and PDE Systems. Birkhauser, 2009.

[10] M. Krstic and A. Smyshlyaev. Backstepping boundary control for first order hyperbolic PDEs and application to systems with actuator and sensor delays. Syst. Contr. Lett., 57:750-758, 2008.

[11] S.J. Moura, N.A. Chaturvedi, and M. Krstic. PDE estimation techniques for advanced battery management systems - Part I: SOC estimation. Proceedings of the 2012 American Control Conference, 2012.

[12] A. Smyshlyaev, E. Cerpa, and M. Krstic. Boundary stabilization of a 1-D wave equation with in-domain antidamping. SIAM J. Control Optim., 48:4014-4031, 2010.

[13] A. Smyshlyaev and M. Krstic. Adaptive Control of Parabolic PDEs. Princeton University Press, 2010.

[14] R. Triggiani. Boundary feedback stabilization of parabolic equations. Appl. Math. Optimiz., 6:201$220,1980$.

[15] R. Vazquez and M. Krstic. Control of Turbulent and Magnetohydrodynamic Channel Flow. Birkhauser, 2008.

[16] R. Vazquez and M. Krstic. Control of 1-D parabolic PDEs with Volterra nonlinearities - Part I: Design. Automatica, 44:2778-2790, 2008.

[17] F. Bribiesca Argomedo, C. Prieur, E. Witrant, and S. Bremond. A Strict Control Lyapunov Function for a Diffusion Equation With Time-Varying Distributed Coefficients. IEEE Trans. Autom. Control, 58:290303, 2013.

[18] R. Vazquez and M. Krstic. Boundary observer for output-feedback stabilization of thermal convection loop. IEEE Trans. Control Syst. Technol., 18:789797, 2010.

[19] R. Vazquez, E. Trelat and J.-M. Coron. Control for fast and stable laminar-to-high-Reynolds-numbers transfer in a 2D Navier-Stokes channel flow. Disc. Cont. Dyn. Syst. Ser. B, 10:925-956, 2008. 